\title{
Baicalein Treatment Promotes Osteoblast Proliferation and Osteogenic Differentiation through Activation of Immediate Early Response 3
}

\author{
Sang-Im Lee ${ }^{\dagger}$ \\ Department of Dental Hygiene, College of Health Sciences, Dankook University, Cheonan 31116, Korea
}

\begin{abstract}
Background: The primary aims of periodontal disease treatment is to remove dental plaque and calculus, the main causes of tooth loss, and restore periodontal tissue destroyed by inflammation. Periodontal disease treatment should also help maintain the alveolar bone, alleviate inflammation, and promote periodontal ligament cell proliferation, which is essential for tissue regeneration. Conventional antibiotics and anti-inflammatories have adverse side effects, especially during long-term use, so there is a need for adjunct treatment agents derived from natural products. The purpose of this study was to investigate whether the herbal flavone baicalein has the osteogenic activity under inflammatory conditions, and assess the involvement of osteoblast immediate early response 3 (IER3) expression.

Methods: Human osteoblastic MG-63 cells were cultured with the pro-inflammatory cytokines tumor necrosis factor $\alpha$ and interleukin $1 \beta$ in the presence and absence of baicalein. Proliferation was assessed using the 3- [4,5-dimethylthiazol-2yl]-2,5-diphenyltetrazolium bromide assay, and expression of IER3 mRNA was assessed using real-time polymerase chain reaction. The expression of IER3 protein levels and activation of associated signal transduction pathways were assessed using western blotting.

Results: Baicalein increased IER3 mRNA and protein expression synergistically. In addition, baicalein reversed the suppression of cell proliferation, and the downregulation of osteogenic transcription factor runt-related transcription factor 2 and osterix induced by pro-inflammatory cytokines. Baicalein also upregulated the phosphorylation of c-Jun N-terminal kinase (JNK) and extracellular signal-regulated kinase (ERK 1/2). The upregulation of IER3 by pro-inflammatory cytokines was blocked by pretreatment with inhibitors of AKT, p38, JNK, and ERK 1/2.

Conclusion: Baicalein mitigates the deleterious responses of osteoblasts to pro-inflammatory cytokines. Further, IER3 enhanced the effect of baicalein via activation of AKT, p38, JNK, and ERK pathways.
\end{abstract}

Key Words: Baicalein, Immediate early response 3 protein, Osteogenesis, Periodontal disease

\section{Introduction}

Periodontal disease and dental caries are two of the most frequent and major diseases of the oral cavity. In the 60 s, more than $90 \%$ of the population suffered from various periodontal diseases. Periodontal disease is caused by bacterial growth due to the presence of food residues in the oral cavity, leading to dental plaque or calculus formation and periodontal inflammation. Chronic inflammation gradually erodes the bone surrounding the teeth, increasing tooth mobility and eventually causing tooth loss ${ }^{1}$. The periodontal ligament located between the tooth cement and the alveolar bone are critical for periodontal regeneration by secreting factors that regulate inflammatory cytokines and the differentiation of local tissue ${ }^{2}$.

Currently, the primary treatment approach for periodontal disease is to physically remove pathogenic bacteria from around the teeth, such as by scaling and root planning. 
However, several variations of bacteria that cause inflammatory reactions are located in the soft tissues or are otherwise anatomically inaccessible, due to which these procedures cannot completely eliminate an inflammatory response $^{3)}$. Therefore, antibiotics and antimicrobial agents are used as auxiliary treatments strategies, but they are restricted because of side effects such as gastrointestinal hypersensitivity and increased antibiotic resistance of bacteria due to long-term use ${ }^{4,5)}$. To overcome these limitations, there has been an increasing interest in identifying natural compounds effective for the prevention and treatment of periodontal disease but with fewer side effects $^{6}$.

In a previous study, we investigated the immunomodulatory and anti-inflammatory activities of various natural products on dental pulp cells and osteoblasts. Resveratrol is a well-known SIRT1 activator, and was responsible for enhancing the lipopolysaccharide- and heat-induced expression of defense genes (hemeoxygenase-1 and human $\beta$-defensin-2) in human dental pulp cells $(\text { HDPCS })^{7)}$. In addition, the expression levels of immune and defense gene against oxidative stress were regulated by ursolic acid ${ }^{8)}$. Furthermore, the plant flavonoid baicalein reportedly promoted angiogenesis along with the odontoblastic differentiation of dental pulp cells ${ }^{9)}$. However, the molecular mechanisms underlying these effects remain unknown.

This study aimed to investigate the protective efficacy of baicalein against the inhibition of osteogenic differentiation induced by pro-inflammatory cytokines and the involvement of immediate early response 3 (IER3) in osteoblastic cells.

\section{Materials and Methods}

\section{Cell culture}

Human osteoblastic MG-63 cells were obtained from the American Type Culture Collection (ATCC, Manassas, VA, USA) and cultured in Dulbecco's modified Eagle medium supplemented with $10 \%$ fetal bovine serum, antibiotics (100 $\mu \mathrm{g} / \mathrm{ml}$ penicillin/streptomycin), and osteogenic supplement $(50 \mu \mathrm{g} / \mathrm{ml}$ ascorbic acid, $10 \mathrm{mM}$ $\beta$-glycerophosphate) at $37^{\circ} \mathrm{C}$ under a humidified atmosphere of $95 \%$ air and $5 \% \mathrm{CO}_{2}{ }^{8}$. All cell culture reagents were obtained from Gibco (Life Technologies, Grand Island, NY, USA). The cells were preincubated for 1 hour with the baicalein (Sigma-Aldrich, St. Louis, MO, USA) or vehicle and then exposed to pro-inflammatory cytokines (10 ng/ml tumor necrosis factor $\alpha[\mathrm{TNF}-\alpha]$ and $10 \mathrm{ng} / \mathrm{ml}$ interleukin $1 \beta$ [IL-1 $\beta$ ] for 24 hours.

\section{Cell proliferation assay}

Cells were plated at $1 \times 10^{4} /$ well in 96-well plates and viable cell number after the indicated treatment was estimated using the conventional 3-[4,5-dimethylthiazol2-yl]-2,5-diphenyltetrazolium bromide (MTT) reduction assay. MTT is a tetrazolium salt cleaved to formazan by the mitochondrial respiratory chain enzyme succinate dehydrogenase in living cells (including osteoblastic MG-63 cells) ${ }^{10)}$. After 24 hours incubation in the indicated condition, the cells were treated with MTT solution (50 $\mu \mathrm{g} / \mathrm{ml}$, Sigma-Aldrich) for 4 hours. The formazan crystals formed were solubilized with dimethyl sulfoxide and the optical density at $540 \mathrm{~nm}$ was measured on a microplate reader as an estimate of the total viable cell number. The MTT assay was performed on independently treated cultures with triplicate internal repeats.

\section{Real-time polymerase chain reaction analysis}

Total RNA was isolated from MG63 cells using TRIzol reagent (Invitrogen, Carlsbad, CA, USA) according to the manufacturer's protocols. RNA quality and concentration were assayed using a NanoDrop 2000 device (Thermo Fisher Scientific, Waltham, MA, USA). cDNA was synthesized from $1 \mu \mathrm{g}$ total RNA and oligo $(\mathrm{dT})_{12 \sim 18}$ primers using AccuPower RT PreMix (Bioneer, Daejeon, Korea). Thereafter, the cDNA $(2 \sim 5 \mu \mathrm{l})$ was amplified with specific primers for glyceraldehyde 3-phosphate dehydrogenase (GAPDH) and IER3 using AccuPower PCR PreMix. Real-time polymerase chain reaction (PCR) assays were performed using SYBR Green premix (Enzynomix, Daejeon, Korea) and a real-time PCR system from Applied Biosystems (Thermo Fisher Scientific). All the primers were purchased from Bioneer. The cycling conditions were: $95^{\circ} \mathrm{C}$ for 20 seconds, $58^{\circ} \mathrm{C}$ for 20 seconds, and $72^{\circ} \mathrm{C}$ for 20 seconds for 32 cycles. The 
Table 1. Real-Time Polymerase Chain Reaction Primers Sequences

\begin{tabular}{lll}
\hline \multicolumn{1}{c}{ Gene } & \multicolumn{1}{c}{ Forward primer (5' -3') $^{\prime}$ ' } & \multicolumn{1}{c}{ Reverse primer (5'-3') } \\
\hline IER3 & 5'-TCTGGTCCCGAGATTTTCAC-3' & 5'-CTCCGAGGTCAGGTTCAAAG-3' \\
GAPDH & 5'-CGGAGTCAACGGATTTGGTCGTAT-3' & 5'-AGCCTTCTCCATGGTGGTGAAGAC-3' \\
\hline
\end{tabular}

IER3: immediate early response 3, GAPDH: glyceraldehyde 3-phosphate dehydrogenase.

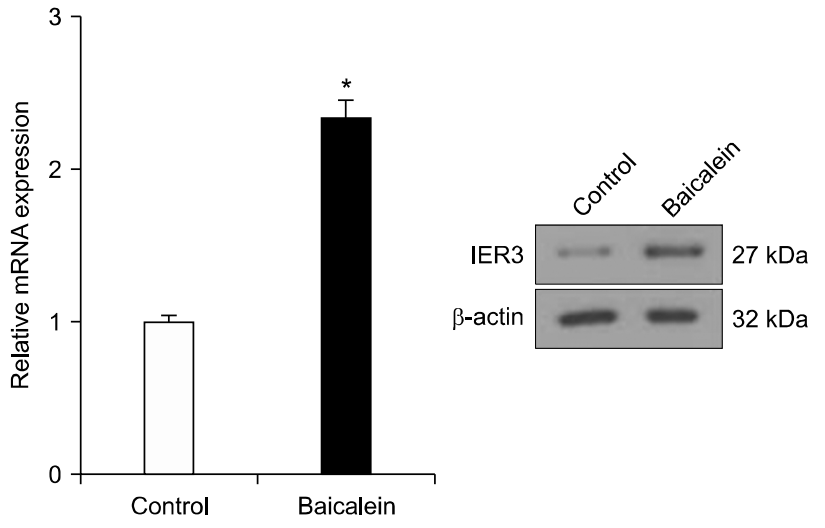

Fig. 1. Effects of baicalein on immediate early response 3 (IER3) expression in human osteoblastic MG-63 cells. Cells were incubated for 24 hours with baicalein $10 \mu \mathrm{M}$. Expression of IER3 was determined using real time polymerase chain reaction (PCR) and Western blot analysis, and representative data of three independent experiments are shown. ${ }^{*} p<0.05$ vs. control.

primer sequences for the genes are shown in Table 1. For quantification, the target gene's expression level was normalized to the GAPDH mRNA level.

\section{Western blot analysis}

Total cell lysates were prepared as described ${ }^{8)}$. Proteins were separated by polyacrylamide gel electrophoresis and transferred onto polyvinylidine difluoride membranes (PVDF; Millipore Co., Milford, MA, USA). Membranes were blotted with primary antibodies (all at 1:1,000 dilution) against the following protein targets using incubation overnight at $4^{\circ} \mathrm{C}$ : AKT and p-AKT (antibodies from Santa Cruz Biotechnology, Dallas, TX, USA); $\beta$-actin and IER3 (antibodies from Abcam, Cambridge, UK), p38, p-p38, c-Jun N-terminal kinase (JNK), p-JNK, extracellular signal-regulated kinase (ERK), and (or) p-ERK (antibodies from Cell Signaling, Danvers, MA, USA). Blots were then incubated with horseradish peroxidase-conjugated secondary antibodies $(1: 2,000)$ for 30 minutes. Proteins were visualized using chemilum- inescence (Amersham Biosciences, Buckinghamshire, UK) emission intensity captured by X-ray film. Image-ProPlus 5.0 software (Media Cybernetics Inc., Rockville, MD, USA) was used for gel densitometric quantification.

\section{Statistical analysis}

All data are presented as mean \pm standard deviation from a minimum of three replicates. Treatment group means were compared to controls using the Student's t-test and the IBM SPSS ver. 25.0 software (IBM Corp., Armonk, NY, USA).

\section{Results}

\section{Baicalein upregulates immediate early} response 3 expression in osteoblasts

To test whether baicalein can upregulate the cellular stress regulator IER3, human osteoblast- like MG-63 cells were treated with non-cytotoxic concentrations $(10 \mu \mathrm{M})$ of baicalein for 24 hours, followed by real-time PCR and western blotting. Indeed, baicalein upregulated IER3 expression in osteoblasts at both mRNA and protein (Fig. 1).

\section{Baicalein increases osteoblast proliferation and osteogenic gene expression}

Osteoblast proliferation is required for osteogenic differentiation, so we evaluated the effects of baicalein on the suppression of MG-63 cell proliferation by proinflammatory cytokines. Proliferation was significantly increased by baicalein treatment for 24 hours compared with that of the control group $(\mathrm{p}<0.05)$, while the proinflammatory cytokine (TNF- $\alpha$ plus IL-1 $\beta$ ) markedly inhibited osteoblast proliferation (Fig. 2A). This suppression of proliferation rate was significantly reversed through 
A

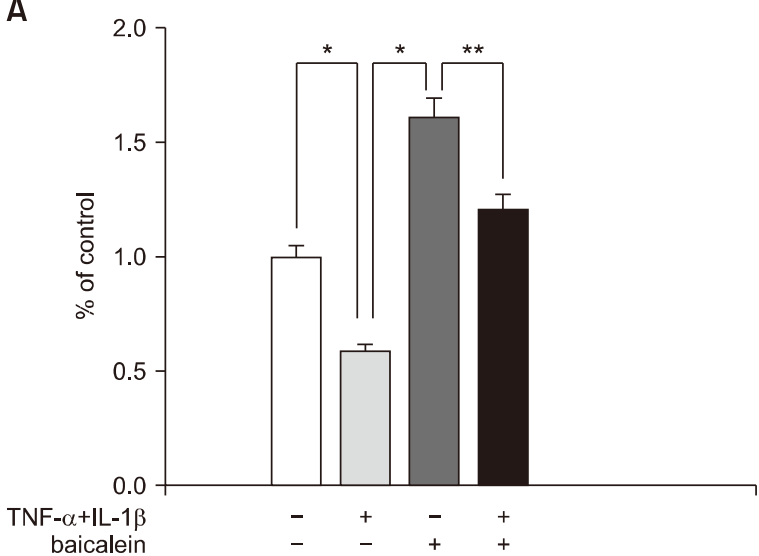

B

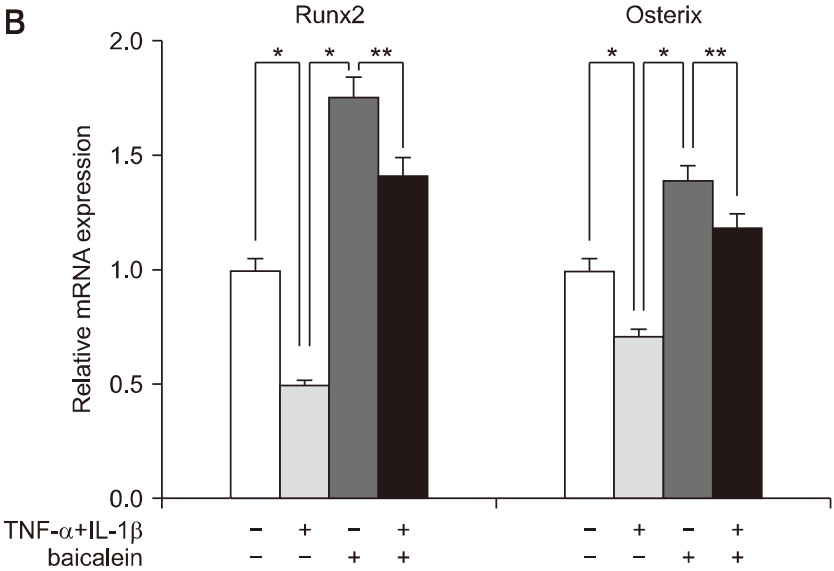

Fig. 2. Effects of baicalein on cytotoxicity and osteogenic differentiation by proinflammatory cytokines in human osteoblastic MG-63 cells. Cells were treated with $10 \mathrm{ng} / \mathrm{ml}$ of tumor necrosis factor $\alpha$ (TNF- $\alpha$ ) plus interleukin $1 \beta$ (IL-1 $\beta$ ) for 24 hours with baicalein 10 $\mu \mathrm{M}$. (A) Proliferation was analyzed using the 3-[4,5-dimethylthiazol-2-yl]-2,5-diphenyltetrazolium bromide assay (A) and expression of runt-related transcription factor 2 (Runx2)/Osterix mRNA using real-time polymerase chain reaction (B) respectively. Values are mean \pm standard deviation of three experiments. ${ }^{*} p<0.05$ vs control and pro-inflammatory cytokines. ${ }^{* *} p<0.05$ vs. baicalein.
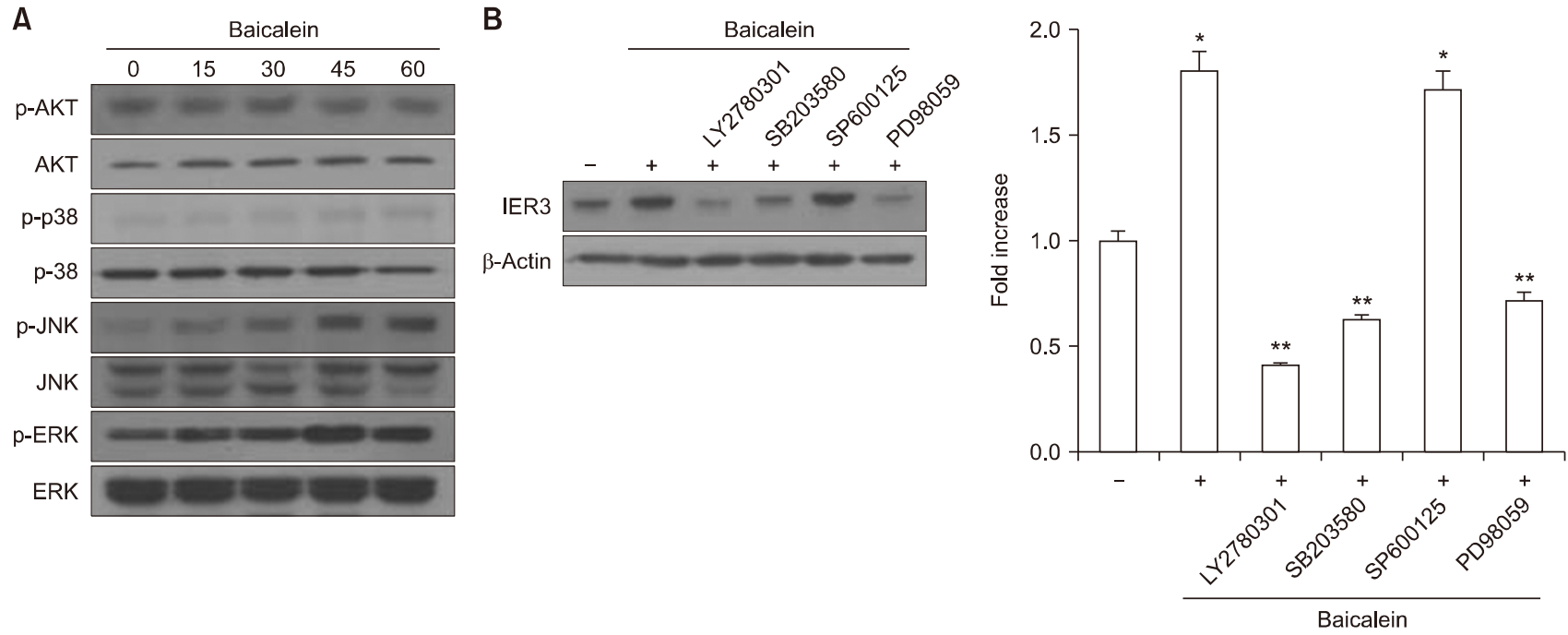

Fig. 3. Effects of various signaling pathway inhibitors on immediate early response 3 (IER3) activation by baicalein. (A) Cell incubated with $10 \mu \mathrm{M}$ baicalein for the indicated times, and then protein expression were examined using Western blot. (B) Cells were pretreated with signal pathway inhibitors $(10 \mu \mathrm{mol} / \mathrm{L}$ of the AKT inhibitor LY2780301, $20 \mu \mathrm{mol} / \mathrm{L}$ of p38 inhibitor SB203580, $20 \mathrm{mmol} / \mathrm{L}$ of the C-Jun $\mathrm{N}$-terminal kinase (JNK) inhibitor SP600125, and $20 \mathrm{mmol} / \mathrm{L}$ of the extracellular signal-regulated kinase (ERK 1/2) inhibitor PD98059 for 1 hour. The data presented are representative of three independent experiments. ${ }^{*} p<0.05$ vs. control. ${ }^{* *} p<0.05$ vs. baicalein.

baicalein co-treatment $(\mathrm{p}<0.05)$. Therefore, baicalein can protect against the reduction in osteogenic differentiation induced by pro-inflammatory cytokines. In osteoblasts, runt-related transcription factor 2 (Runx2) and osterix are crucial transcription factors for the initiation and regulation of early osteogenesis. Consistent with the protection of osteogenesis, baicalein reversed the inhibitory effects of TNF- $\alpha$ plus IL- $1 \beta$ on Runx 2 and osterix mRNA expression levels (Fig. 2B).

\section{Immediate early response 3 mediates} baicalein-induced osteogenic differentiation

To investigate the signaling pathways mediating IER3 upregulation by baicalein, we examined the phosphoactivation status of AKT, p38, JNK, and p42/44 (ERK 1/2) mitogen-activated protein kinase (MAPK) proteins and the effects of various pathway inhibitors on the IER3 protein levels following baicalein treatment. As shown in Fig. 3A, treatment with baicalein improved the pho- 
sphorylation levels of JNK and ERK $1 / 2$ in a timedependent manner, but had no effects on AKT and p38 phosphorylation status. Pretreatment of cells with the AKT inhibitor LY2780301, p38 inhibitor SB203580, and ERK 1/2 inhibitor PD98059 significantly blocked baicaleininduced expression of IER3 protein, in contrast the JNK inhibitor SP600125 was ineffective (Fig. 3B).

\section{Discussion}

Recent studies have reported a trend toward more frequent use of natural compounds including baicalein for oral healthcare ${ }^{11)}$. Baicalein is one of the major flavonoids in Scutellaria baicalensis Georgi, which has been used in Asia as a herbal medicine. Several biological effects of baicalein, including antioxidant and anti-inflammatory activities $^{12,13)}$, have been reported. Recently several studies have demonstrated that baicalein alone has antimicrobial activities against oral bacteria, and can potentiate the effects of antibiotics ${ }^{14}$. We previously reported that the baicalein promoted angiogenesis and odontoblastic differentiation via the BMP and Wnt pathways in $\mathrm{HDPCS}^{9)}$. Additionally, baicalein stimulated MC3T3-E1 cells to differentiate via the osteoblasts through activation of the rapamycin complex 1 signaling pathway, including protein kinases and transcription factors such as phospho-4E (eIF4E)-binding protein 1 and phospho-p70 ribosomal S6 kinase $1^{15}$ ). Although other lower doses of baicalein treatments did not affect osteoblast cell viability, baicalein suppressed the differentiation of osteoblastic cell line MC3T3-E1 ${ }^{16)}$. However, we observed that a low-dose of baicalein $(10 \mu \mathrm{M})$ reversed the suppression of osteogenic transcription factor expression (Runx2 and osterix) induced by TNF- $\alpha$ plus IL- $1 \beta$ stimulation.

IER3, also known as IEX-1, Dif-2, gly96, or p22/ PRG-1, is rapidly induced by a wide range of stimuli, including growth factors, cytokines, DNA damage, and viral infection ${ }^{17)}$. In the human genome, IER3 does not have other close homologues, and its sequence is well conserved among mammals. IER3 mRNA is widely expressed in most human tissues, with abundant expression in the epithelial tissues with high cell turnovers ${ }^{18)}$. Recent studies have suggested that IER3 is upregulated in human periodontal ligament cells under cyclic tensile stress ${ }^{19)}$. IER3 is known to be involved in cellular processes, including apoptosis, proliferation, differentiation, and DNA repair under cellular stress ${ }^{20)}$. Our reported showed that osteoblast proliferation was markedly inhibited by pro-inflammatory cytokine (TNF- $\alpha$ plus IL-1 $\beta$ ), and then this suppression of proliferation rate was significantly reversed through baicalein co-treatment. Therefore, baicalein demonstrated that it can protect against the reduction in osteogenic differentiation induced by proinflammatory cytokines.

Baicalein has reportedly induced the activation of MAPK and transcription factor signaling pathway, and activated the NF- $\kappa \mathrm{B}$ in the early stages of osteoblast differentiation $^{21)}$. The AKT pathways, JNK, ERK 1/2, and p38 MAPK were activated in the inflammatory conditions of several cell types. The present study demonstrated that baicalein activated JNK and ERK 1/2 signaling pathways, unlike AKT and p-38. Moreover, IER3 upregulation was associated with baicalein-induced osteogenic differentiation of osteoblast and that these processes were regulated via the AKT and MAPK signaling pathway.

In conclusion, the present study demonstrated that baicalein promoted osteoblastic differentiation via activation of IER3. Baicalein has an osteogenic enhancing effects in inflammatory microenvironment, along with a regulatory effect with regard to IER3 activation via AKT and ERK $1 / 2$ pathways. These findings provide additional evidence for the potential clinical efficacy of baicalein against periodontal inflammation.

\section{Notes}

\section{Conflict of interest}

No potential conflict of interest relevant to this article was reported.

\section{Ethical approval}

This project does not require IRB review because it is an experimental paper using commercially available cells.

\section{ORCID}

Sang-Im Lee, https://orcid.org/0000-0003-2635-6876 


\section{Acknowledgements}

This research was supported by the National Research Foundation of Korea (NRF) grant funded by the Korea government (MSIP) (No. 2018R1D1A1B07051253).

\section{References}

1. Pihlstrom BL, Michalowicz BS, Johnson NW: Periodontal diseases. Lancet 366: 1809-1820, 2005. https://doi.org/10.1016/S0140-6736(05)67728-8

2. Lee SI, Yu JS: NFATc Mediates lipopolysaccharide and nicotine-induced expression of iNOS and COX-2 in human periodontal ligament cells. J Dent Hyg Sci 15: 753-760, 2015. https://doi.org/10.17135/jdhs.2015.15.6.753

3. Christersson LA, Albini B, Zambon JJ, Wikesjö UM, Genco RJ: Tissue localization of Actinobacillus actinomycetemcomitans in human periodontitis. I. Light, immunofluorescence and electron microscopic studies. J Periodontol 58: 529-539, 1987. https://doi.org/10.1902/jop.1987.58.8.529

4. Serino G, Rosling B, Ramberg P, Hellström MK, Socransky SS, Lindhe J: The effect of systemic antibiotics in the treatment of patients with recurrent periodontitis. J Clin Periodontol 28: 411-418, 2001. https://doi.org/10.1034/j.1600-051x.2001.028005411.x

5. Forner L, Nielsen CH, Bendtzen K, Larsen T, Holmstrup P: Increased plasma levels of IL-6 in bacteremic periodontis patients after scaling. J Clin Periodontol 33: 724-729, 2006. https://doi.org/ \10.1111/j.1600-051X.2006.00964.X

6. Kouidhi B, Al Qurashi YM, Chaieb K: Drug resistance of bacterial dental biofilm and the potential use of natural compounds as alternative for prevention and treatment. Microb Pathog 80: 39-49, 2015.

https://doi.org/10.1016/j.micpath.2015.02.007

7. Lee SI, Min KS, Bae WJ, et al.: Role of SIRT1 in heat stressand lipopolysaccharide-induced immune and defense gene expression in human dental pulp cells. J Endod 37: 1525-1530, 2011. https://doi.org/10.1016/j.joen.2011.07.006

8. Lee SI: Protective effects of ursolic acid on osteoblastic differentiation via activation of IER3/Nrf2. J Dent Hyg Sci 19: 198-204, 2019. https://doi.org/10.17135/jdhs.2019.19.3.198

9. Lee SI, Kim SY, Park KR, Kim EC: Baicalein promotes angiogenesis and odontoblastic differentiation via the BMP and Wnt pathways in human dental pulp cells. Am J Chin Med 44: 1457-1472, 2016. https://doi.org/10.1142/S0192415X16500816

10. Mosmann T: Rapid colorimetric assay for cellular growth and survival: application to proliferation and cytotoxicity assays. J Immunol Methods 65: 55-63, 1983. https://doi.org/10.1016/0022-1759(83)90303-4

11. Narotzki B, Reznick AZ, Aizenbud D, Levy Y: Green tea: a promising natural product in oral health. Arch Oral Biol 57: 429-435, 2012. https://doi.org/10.1016/j.archoralbio.2011.11.017

12. Jang EJ, Cha SM, Choi SM, Cha JD: Combination effects of baicalein with antibiotics against oral pathogens. Arch Oral Biol 59: 1233-1241, 2014. https://doi.org/10.1016/j.archoralbio.2014.07.008

13. Li X, Luo W, Ng TW, et al.: Nanoparticle-encapsulated baicalein markedly modulates pro-inflammatory response in gingival epithelial cells. Nanoscale 9: 12897-12907, 2017. https://doi.org/10.1039/c7nr02546g

14. Dai C, Tang S, Wang Y, Velkov T, Xiao X: Baicalein acts as a nephroprotectant that ameliorates colistin-induced nephrotoxicity by activating the antioxidant defence mechanism of the kidneys and down-regulating the inflammatory response. J Antimicrob Chemother 72: 2562-2569, 2017. https://doi.org/10.1093/jac/dkx185

15. Li SF, Tang JJ, Chen J, et al.: Regulation of bone formation by baicalein via the mTORC1 pathway. Drug Des Devel Ther 9: 5169-5183, 2015. https://doi.org/10.2147/DDDT.S81578

16. Lai $\mathrm{CH}, \mathrm{Wu} \mathrm{YW}$, Yeh SD, Lin YH, Tsai YH: Effects of 6-hydroxyflavone on osteoblast differentiation in MC3T3-E1 cells. Evid Based Complement Alternat Med 2014: 924560, 2014. https://doi.org/10.1155/2014/924560

17. Arlt A, Schäfer H: Role of the immediate early response 3 (IER3) gene in cellular stress response, inflammation and tumorigenesis. Eur J Cell Biol 90: 545-552, 2011. https://doi.org/10.1016/j.ejcb.2010.10.002

18. Kumar R, Kobayashi T, Warner GM, et al.: A novel immediate early response gene, IEX-1, is induced by ultraviolet radiation in human keratinocytes. Biochem Biophys Res Commun 253: 336-341, 1998. https://doi.org/10.1006/bbrc.1998.9692

19. Wang Y, Li Y, Fan X, Zhang Y, Wu J, Zhao Z: Early proliferation alteration and differential gene expression in 
human periodontal ligament cells subjected to cyclic tensile stress. Arch Oral Biol 56: 177-186, 2011.

https://doi.org/10.1016/j.archoralbio.2010.09.009

20. Jin H, Suh DS, Kim TH, Yeom JH, Lee K, Bae J: IER3 is a crucial mediator of TAp73 $\beta$-induced apoptosis in cervical cancer and confers etoposide sensitivity. Sci Rep 5: 8367,
2015. https://doi.org/10.1038/srep08367

21. Kim JM, Lee SU, Kim YS, Min YK, Kim SH: Baicalein stimulates osteoblast differentiation via coordinating activation of MAP kinases and transcription factors. J Cell Biochem 104: 1906-1917, 2008. https://doi.org/10.1002/jcb.21760 